\title{
Boundary Stabilization of Underground Multirobot System with Virtual String Constraints
}

\author{
Lingling Su, ${ }^{1}$ Xianhua Zheng, ${ }^{2}$ Yongshi Song, ${ }^{2}$ Shang Feng, ${ }^{3}$ and Lin Zhang $\mathbb{D}^{2}$ \\ ${ }^{1}$ College of Science, North China University of Technology, Beijing, China \\ ${ }^{2}$ School of Robot Engineering, Yangtze Normal University, Chongqing, China \\ ${ }^{3}$ Faculty of Computing, Harbin Institute of Technology, Harbin, China \\ Correspondence should be addressed to Lin Zhang; lin.zhang_2014@hotmail.com
}

Received 16 December 2021; Accepted 11 January 2022; Published 10 February 2022

Academic Editor: Phang Chang

Copyright (C 2022 Lingling Su et al. This is an open access article distributed under the Creative Commons Attribution License, which permits unrestricted use, distribution, and reproduction in any medium, provided the original work is properly cited.

The manipulation of multiple physical connected objects can be described by virtual connected string system. We proposed a Lyapunov-based stabilization control approach for virtual connected string with midway discontinue vertical force. The system is with Riemannian boundary. We use the backstepping method to transform the system into a stable system with Dirichlet boundary. In this way, we get the controller to make sure the closed-loop system converge to the zero point exponentially. Then, we construct a Lyapunov function to analyze the stability for the closed-loop system. We also show the system is well-posedness. Also, we use the active disturbance rejection control (ADRC) to reject the disturbance when disturbance is present. Some numerical stimulations show that the control law is the effective.

\section{Introduction}

Multirobot transportation system has been one of the most potential research fields in recent years, and the majority of these studies are focused on multiple robots manipulating a single object, while the manipulated object in this paper is a class of multiple physical connected objects (MPO). The manipulation of MPO has been widely used both in daily life and industrial application. As an example, flood shield is used to obstruct flood disaster. These shields are connected with each other, and each shield is immobilized by fixed oblique support, flexible oblique support, or vertical support, which are represented in Figures 1(a)-1(c), respectively. Retaining wall is another typical application of MPO, i.e., retaining mesh, which is supported by multiple linked rods, as shown in Figure 1(d). One more abstracted application, a multiple link model-based human body is held by two-arm service robot, as shown in Figure 1(e). In this application, human beings are abstracted as free-ended multiple connected rigid bodies, and these rigid bodies are manipulated by two arms, respectively. Similar to above applications, the pushing manipulation problem of mobile support robot
(MSR, also known as hydraulic support or roof support) on underground fully mechanized coal mining face is a such kind of system as well. Since the manipulated object, i.e., scraper conveyor, is composed of multiple middle troughs (MTs), we simplified scraper conveyor as multiple connected objects with physical connections. As shown in Figures 1(f) and $1(\mathrm{~g})$, both the physical and simulated workplace of multiple MTs manipulated by MSRs on ground is represented, respectively. With simplification, as shown in Figure $1(\mathrm{~h})$, MPO is replaced by external virtual string. Then, the simulated external constraint using virtual connected string is presented, as shown in Figure 1(i).

Due to loadable characteristics as well as its mobility through pushing and advancing, the manipulation problem of MSRs is one of the most complicated problems on underground fully mechanized coal mining face. Based on our previous research on the underground multirobot system $[1,2]$, we found the main difficulty is modeling the external constraints. Considering the commonalities of above applications, a reasonable assumption is proposed by replacing MPO with virtual connected string constraints, and the main problem is boundary stabilization of virtual connected string 

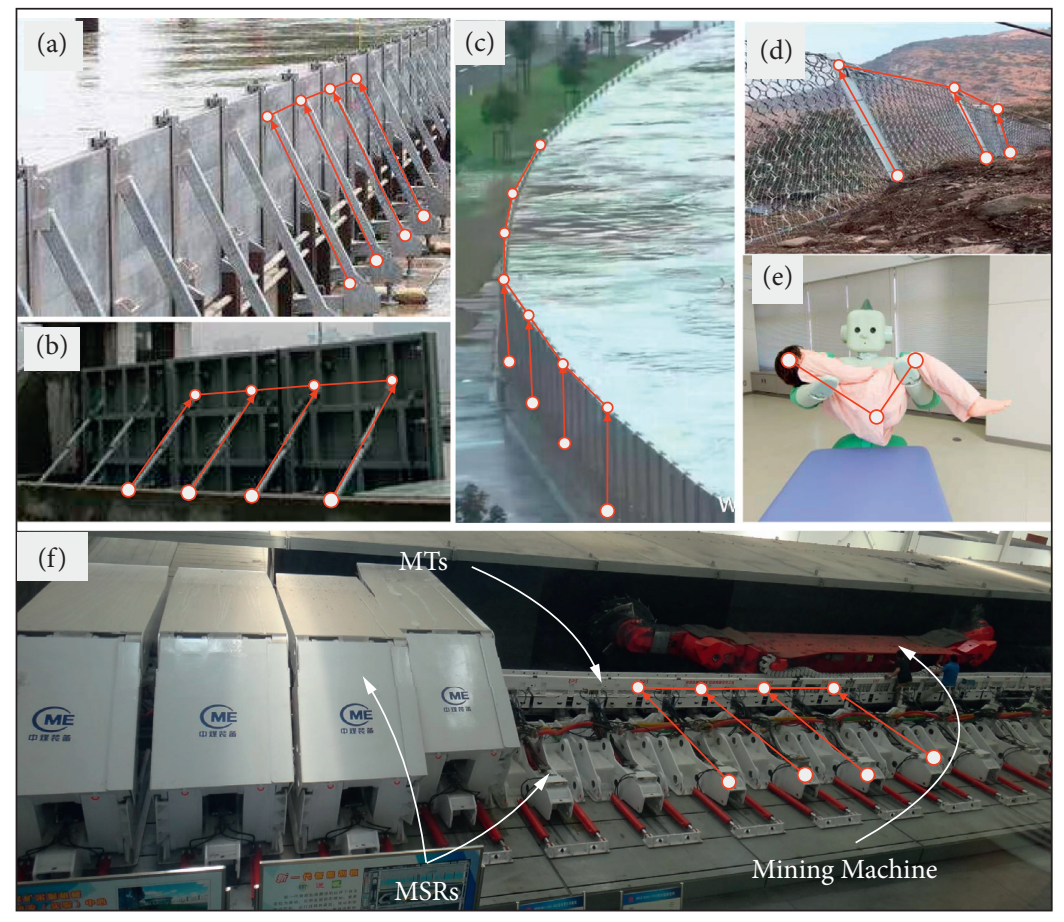

(g) Multi-robot system with MPO

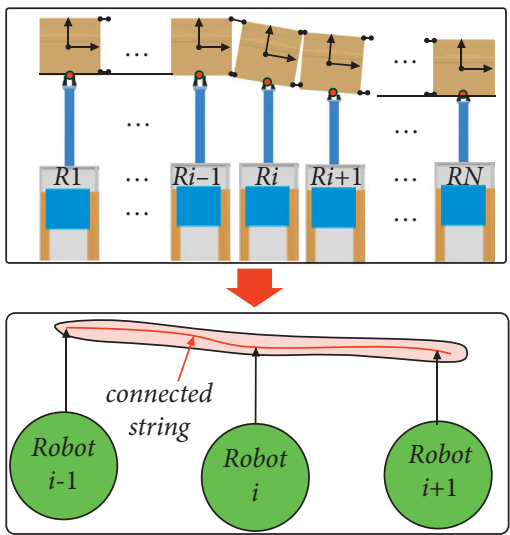

(h) Replace MPO with external string

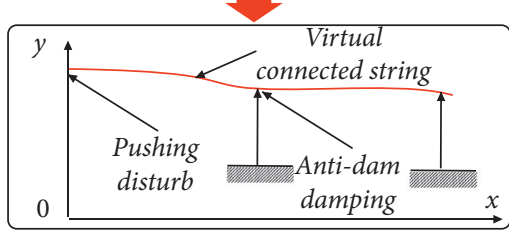

(i) Simulate external constraints using virtual connected string

FIGURE 1: Widely applied scenarios of MPO. (a) Flood shield immobilized by multiple fixed oblique supports. (b) Flood shield immobilized by multiple flexible oblique supports. (c) Flood shield immobilized by multiple vertical supports. (d) Retaining wall supported by multiple bars. (e) Multiple link model-based person held by the two-arm service robot. (f) The simulated workplace of multiple MTs manipulated by MSRs on the ground. The scraper conveyor is composed of many middle troughs (MTs) and each MT is connected with other. MSRs push corresponding MT forward. (g) The diagram of the multirobot system with MPO. (h) Generalized system architecture of the multirobot system, whose manipulating object is replaced with virtual external string. (i) Simulated external constraints through virtual connected string, and both the disturbance and antidamping are presented.

system. Thus, one contribution of this paper is replacing the manipulated MPO with a virtual connected string. Then, the problem is converted to the system stabilization of the connected string system considering pushing disturbance and antidamping.

The study for the virtual connected string equations has drawn significant attention in recent years. It can be applied widely in engineering field. For example, aerial cable, traffic problem, and transmission lines can be described by connected string systems $[3,4]$. In [5], the stability of strings with various connected feedbacks is studied by spectral analysis. In [2], the control of a connected string converter modeled from electrical power is studied. In [6], the symmetry is considered for the system of strings. Other literature relevant can be found in [7-9] and the references therein.
Although there are some works on the stability of connected strings, there is still no result on the Lyapunov stability analysis for connected strings. It needs some mathematical techniques to find the suitable Lyapunov function for connected strings. Moreover, these models are without disturbance. However, disturbance is usually present in practical applications [10-12]. So, we are also concerned with the disturbance for the model. Sliding mode control and active disturbance rejection control (ADRC) are usually used to reject the disturbance [13-19]. ADRC is used in this paper. ADRC shows its advantages because it can not only estimate the disturbance but also counteract the disturbance (see [20-24]).

We consider a connected string system, which is connected at point $x=1$ in $[0,2]$ :

$$
\begin{cases}\chi_{t t}(x, t)=\chi_{x x}(x, t), & x \in(0,1) \cup(1,2), t>0, \\ \chi\left(1^{-}, t\right)=\chi\left(1^{+}, t\right), & t \geq 0, \\ \chi_{x}\left(1^{-}, t\right)-\chi_{x}\left(1^{+}, t\right)=q \chi_{t}(1, t), & t \geq 0, \\ \chi_{x}(0, t)=U(t)+r(t), & t \geq 0, \\ \chi_{x}(2, t)=0, & t \geq 0,\end{cases}
$$


where $\chi$ is the state, $U(t)$ is the input, $q>0, q \neq 2$, and $r$ is the disturbance; we also assume $|r(t)| \leq M,|\dot{r}(t)| \leq M, \forall t>0$. The connected term is the discontinuity of vertical force component; it is antistable.

We introduce a new variable $\varphi(x, t)=\left[\varphi_{1}(x, t)\right.$, $\left.\varphi_{2}(x, t)\right]^{T}$, where

$$
\varphi_{1}(x, t)=\chi(x, t), \varphi_{2}(x, t)=\chi(2-x, t), x \in[0,1], t \geq 0 .
$$

Then, system (1) becomes

$$
\begin{cases}\varphi_{t t}(x, t)=\varphi_{x x}(x, t), & 0<x<1, t>0, \\ \varphi_{1}(1, t)=\varphi_{2}(1, t), & t \geq 0, \\ \varphi_{1 x}(1, t)+\varphi_{2 x}(1, t)=q \varphi_{1 t}(1, t), & t \geq 0, \\ \varphi_{1 x}(0, t)=U(t)+r(t), & t \geq 0, \\ \varphi_{2 x}(0, t)=0, & t \geq 0 .\end{cases}
$$

$$
\begin{aligned}
\theta_{1}(x, t)= & \varphi_{1}(x, t)+\frac{q(q+c)}{4-q^{2}}\left[\varphi_{1}(x, t)+\varphi_{2}(x, t)\right]-\frac{q(q+c)}{4-q^{2}}\left[\varphi_{1}(1, t)+\varphi_{2}(1, t)\right] \\
& +\frac{2(q+c)}{4-q^{2}} \int_{x}^{1}\left[\varphi_{1 t}(y, t)+\varphi_{2 t}(y, t)\right] \mathrm{d} y, \\
\theta_{2}(x, t)= & \varphi_{2}(x, t),
\end{aligned}
$$

where $q>0, q \neq 2$ and $c>0, c \neq 2$; its inverse transformation is

$$
\begin{aligned}
\varphi_{1}(x, t)= & \theta_{1}(x, t)+\frac{q(q+c)}{4-c^{2}}\left[\theta_{1}(x, t)+\theta_{2}(x, t)\right]-\frac{q(q+c)}{4-c^{2}}\left[\theta_{1}(1, t)+\theta_{2}(1, t)\right] \\
& +\frac{2(q+c)}{4-c^{2}} \int_{x}^{1}\left[\theta_{1 t}(y, t)+\theta_{2 t}(y, t)\right] \mathrm{d} y, \\
\varphi_{2}(x, t)= & \theta_{2}(x, t) .
\end{aligned}
$$

By transformation (4) and (5), system (3) is going to be

$$
\begin{cases}\theta_{t t}(x, t)=\theta_{x x}(x, t), & 0<x<1, \quad t>0, \\ \theta_{1}(1, t)=\theta_{2}(1, t), & t \geq 0, \\ \theta_{1 x}(1, t)+\theta_{2 x}(1, t)=-c \theta_{1 t}(1, t), & t \geq 0, \\ \theta_{1 x}(0, t)=\frac{4-c^{2}}{4+q c}(U(t)+r(t))-\frac{2(q+c)}{4+q c}\left(\theta_{1 t}(0, t)+\theta_{2 t}(0, t)\right), & t \geq 0, \\ \theta_{2 x}(0, t)=0, & t \geq 0 .\end{cases}
$$


By the transformation, the connected antidamping is moved to the boundary. We give a controller:

$$
\begin{aligned}
U_{1}(t)= & \frac{2(q+c)}{4-c^{2}}\left[\theta_{1 t}(0, t)+\theta_{2 t}(0, t)\right] \\
& +k \frac{4+q c}{4-c^{2}} \theta_{1}(0, t), k>0,
\end{aligned}
$$

to deal with the antidamping term at point $x=0$. Under the control of

$$
U(t)=U_{0}(t)+U_{1}(t)
$$

where $U_{0}(t)$ is a new control to be designed to contract the disturbance. System (7) becomes

$$
\begin{cases}\theta_{t t}(x, t)=\theta_{x x}(x, t), & 0<x<1, t>0, \\ \theta_{1}(1, t)=\theta_{2}(1, t), & t \geq 0, \\ \theta_{1 x}(1, t)+\theta_{2 x}(1, t)=-c \theta_{1 t}(1, t), & t \geq 0, \\ \theta_{1 x}(0, t)=\frac{4-c^{2}}{4+q c}\left(U_{0}(t)+r(t)\right)+k \theta_{1}(0, t), & t \geq 0, \\ \theta_{2 x}(0, t)=0, & t \geq 0 .\end{cases}
$$

We consider system (10) in the Hilbert space $\mathscr{H}=\left\{\left(u_{1}, v_{1}, u_{2}, v_{2}\right) \in\left(H^{1}(0,1) \times L^{2}(0,1)\right)^{2} \mid u_{1}(1)=u_{2}(1)\right\}$.
With the inner product induced norm, for $\forall\left(u_{1}, v_{1}, u_{2}, v_{2}\right) \in \mathscr{H}$,

$$
\|\left.\left(u_{1}, v_{1}, u_{2}, v_{2}\right)\left|=\int_{0}^{1}\left(\left|u_{1}^{\prime}(x)\right|^{2}+\left|v_{1}(x)\right|^{2}+\left|u_{2}^{\prime}(x)\right|^{2}+\left|v_{2}(x)\right|^{2}\right) \mathrm{d} x+k\right| u_{1}(0)\right|^{2}
$$

Introduce an unbounded linear operator $\mathscr{A}: D(\mathscr{A})(\subset \mathscr{H}) \longrightarrow \mathscr{H}:$

$$
\left\{\begin{array}{l}
\mathscr{A} Z=\left(v_{1}, u_{1}^{\prime \prime}, v_{2}, u_{2}^{\prime \prime}\right), \forall Z=\left(u_{1}, v_{1}, u_{2}, v_{2}\right) \in D(\mathscr{A}) \\
D(\mathscr{A})=\left\{Z \in H \cap\left(H^{2}(0,1) \times H^{1}(0,1)\right)^{2} \mid u_{1}^{\prime}(1)+u_{2}^{\prime}(1)=-c v_{1}(1)\right. \\
u_{1}^{\prime}(0)=k u_{1}(0) \\
u_{2}^{\prime}(0)=0 \\
v_{1}(1)=v_{2}(1)
\end{array}\right.
$$

and then, system (10) can be written in the evolution equation form in $\mathscr{H}$ :

$$
\left\{\begin{array}{l}
\frac{\mathrm{d}}{\mathrm{d} t} Z(t)=\mathscr{A}\left(\theta_{1}(\cdot, t), \theta_{1 t}(\cdot, t), \theta_{2}(\cdot, t), \theta_{2 t}(\cdot, t)\right)-\frac{4-c^{2}}{4+q c} B\left(U_{0}(t)+r(t)\right) \\
Z(\cdot, 0)=\left(\theta_{1}(\cdot, 0), \theta_{1 t}(\cdot, 0), \theta_{2}(\cdot, 0), \theta_{2 t}(\cdot, 0)\right),
\end{array}\right.
$$


where $Z(t)=\left(\theta_{1}(\cdot, t), \theta_{1 t}(\cdot, t), \theta_{2}(\cdot, t), \theta_{2 t}(\cdot, t)\right)$ and $\mathscr{B}=$ $(0, \delta(x), 0,0)$. We get the following two lemmas.
Proof. We will first demonstrate that $\mathscr{A}$ generates a $\mathrm{C}_{0}$-semigroup. A direct computation gives

Lemma 1. A generates an exponentially stable $\mathrm{C}_{0}$-semigroup.

$$
\begin{aligned}
\operatorname{Re} & \mathscr{A}\left(u_{1}, v_{1}, u_{2}, v_{2}\right)^{T},\left(u_{1}, v_{1}, u_{2}, v_{2}\right)^{T} \\
& =\int_{0}^{1}\left(v_{1}^{\prime}(x, t) u_{1}^{\prime \prime}(x, t)+u_{1}^{\prime \prime}(x, t) v_{1}(x, t)+v_{2}^{\prime}(x, t) u_{2}^{\prime \prime}(x, t)+u_{2}^{\prime \prime}(x, t) v_{2}(x, t)\right) \mathrm{d} x+k v_{1}(0) u_{1}(0) \\
& =-c\left|v_{1}(1)\right|^{2} \leq 0 .
\end{aligned}
$$

So, $\mathscr{A}$ is dissipative.

For any given $\left(\phi_{1}, \varphi_{1}, \phi_{2}, \varphi_{2}\right) \in \mathscr{H}$, solve $\mathscr{A}\left(u_{1}, v_{1}, u_{2}, v_{2}\right)$ $=\left(\phi_{1}, \varphi_{1}, \phi_{2}, \varphi_{2}\right)$, to obtain

$$
\left\{\begin{array}{l}
v_{1}(x)=\phi_{1}(x), \\
v_{2}(x)=\phi_{2}(x),
\end{array}\right.
$$

which gives

$$
\left\{\begin{array}{l}
u_{1}(x)=(k x+1) u_{1}(0)+\int_{0}^{x} \varphi_{1}(\eta)(x-\eta) d \eta \\
u_{2}(x)=u_{2}(0)+\int_{0}^{x} \varphi_{2}(\eta)(x-\eta) d \eta \\
u_{1}(0)=-\frac{1}{k}\left[\int_{0}^{1}\left(\varphi_{1}(\eta)+\varphi_{2}(\eta)\right) d \eta+c v_{1}(1)\right] \\
u_{2}(0)=(k+1) u_{1}(0)+\int_{0}^{1} \varphi_{1}(\eta)(1-\eta) d \eta-\int_{0}^{1} \varphi_{2}(\eta)(1-\eta) d \eta
\end{array}\right.
$$

So, $\left(u_{1}, v_{1}, u_{2}, v_{2}\right) \in \mathscr{H}$ is unique. Hence, $\mathscr{A}^{-1}$ exists, and from the Sobolev embedding theorem, it is also compact on $\mathscr{H}$. Using Lumer-Phillips theorem (see [28]), $\mathscr{A}$ generates a $C_{0}$-semigroup of contractions $e^{\mathscr{A} t}$.

Next, we show that $\mathscr{A}$ is exponentially stable. We consider the evolution equation:

$$
\frac{\mathrm{d}}{\mathrm{d} t} Z(t)=\mathscr{A} Z(t), Z(t)=\left(\theta_{1}(\cdot, t), \theta_{1 t}(\cdot, t), \theta_{2}(\cdot, t), \theta_{2 t}(\cdot, t)\right),
$$

which equals to the following PDE:

$$
\begin{cases}\theta_{t t}(x, t)=\theta_{x x}(x, t), & 0<x<1, t>0, \\ \theta_{1}(1, t)=\theta_{2}(1, t), & t \geq 0, \\ \theta_{1 x}(1, t)+\theta_{2 x}(1, t)=-c \theta_{1 t}(1, t) & t \geq 0, \\ \theta_{1 x}(0, t)=k \theta_{1}(0, t), & t \geq 0, \\ \theta_{2 x}(0, t)=0 & t \geq 0 .\end{cases}
$$

Let $V(t)$ be a function given by

$$
\begin{aligned}
V(t)= & \frac{1}{2} \int_{0}^{1}\left(\theta_{1 x}^{2}(x, t)+\theta_{1 t}^{2}(x, t)+\theta_{2 x}^{2}(x, t)+\theta_{2 t}^{2}(x, t)\right) \mathrm{d} x+\frac{1}{2} k \theta_{1}^{2}(0, t) \\
& +\delta \int_{0}^{1}\left[\theta_{1 x}(x, t) \theta_{1 t}(x, t)+\theta_{2 x}(x, t) \theta_{2 t}(x, t)\right] \mathrm{d} x \\
& +\delta \int_{0}^{1}\left[\theta_{1 t}(x, t) \theta_{2 x}(x, t)+\theta_{2 t}(x, t) \theta_{1 x}(x, t)\right] \mathrm{d} x,
\end{aligned}
$$

where $\delta>0$ and $\delta$ is sufficiently small. 
From Cauchy-Schwartz and Young's inequalities, it is found that, for $\delta \leq \min \{1 / 3, k / 3\}$, there exists $m_{1}, m_{2}>0$ so that $m_{1}\|Z(t)\|^{2} \leq V(t) \leq m_{2}\|Z(t)\|^{2}$, where

$$
\begin{aligned}
& m_{1}=\min \left\{\frac{1-3 \delta}{2}, \frac{k-3 \delta}{2}\right\}, \\
& m_{2}=\max \left\{\frac{1+3 \delta}{2}, \frac{k+3 \delta}{2}\right\},
\end{aligned}
$$

$\|Z(t)\|^{2}=\left\|\theta_{1 x}\right\|^{2}+\left\|\theta_{1 t}\right\|^{2}+\left\|\theta_{1}(0)\right\|^{2}+\left\|\theta_{2 x}\right\|^{2}+\left\|\theta_{2 t}\right\|^{2}$.
Hence, $V(t)$ is a proper Lyapunov function for (23). By taking the deviation of $V(t)$ in $t$, we have

$$
\begin{aligned}
& \dot{V}(t)=\int_{0}^{1}\left[\theta_{1 x}(x, t) \theta_{1 x t}(x, t)+\theta_{1 t}(x, t) \theta_{1 t t}(x, t)+\theta_{2 x}(x, t) \theta_{2 x t}(x, t)+\theta_{2 t}(x, t) \theta_{2 t t}(x, t)\right] \mathrm{d} x \\
& +k \theta_{1}(0, t) \theta_{1 t}(0, t)+\delta \int_{0}^{1}\left[\theta_{1 x t}(x, t) \theta_{1 t}(x, t)+\theta_{1 x}(x, t) \theta_{1 t t}(x, t)+\theta_{2 x t}(x, t) \theta_{2 t}(x, t)+\theta_{2 x}(x, t) \theta_{2 t t}(x, t)\right] \mathrm{d} x \\
& +\delta \int_{0}^{1}\left[\theta_{1 t t}(x, t) \theta_{2 x}(x, t)+\theta_{1 t}(x, t) \theta_{2 x t}(x, t)+\theta_{2 t t}(x, t) \theta_{1 x}(x, t)+\theta_{2 t}(x, t) \theta_{1 x t}(x, t)\right] \mathrm{d} x, \\
& =\int_{0}^{1}\left[\theta_{1 x}(x, t) \theta_{1 x t}(x, t)+\theta_{1 t}(x, t) \theta_{1 x x}(x, t)+\theta_{2 x}(x, t) \theta_{2 x t}(x, t)+\theta_{2 t}(x, t) \theta_{2 x x}(x, t)\right] \mathrm{d} x \\
& +k \theta_{1}(0, t) \theta_{1 t}(0, t)+\delta \int_{0}^{1}\left[\theta_{1 x t}(x, t) \theta_{1 t}(x, t)+\theta_{1 x}(x, t) \theta_{1 x x}(x, t)+\theta_{2 x t}(x, t) \theta_{2 t}(x, t)+\theta_{2 x}(x, t) \theta_{2 x x}(x, t)\right] \mathrm{d} x \\
& +\delta \int_{0}^{1}\left[\theta_{1 x x}(x, t) \theta_{2 x}(x, t)+\theta_{1 t}(x, t) \theta_{2 x t}(x, t)+\theta_{2 x x}(x, t) \theta_{1 x}(x, t)+\theta_{2 t}(x, t) \theta_{1 x t}(x, t)\right] \mathrm{d} x, \\
& =\theta_{1 t}(1, t) \theta_{1 x}(1, t)-\theta_{1 t}(0, t) \theta_{1 x}(0, t)+\theta_{2 t}(1, t) \theta_{2 x}(1, t)-\theta_{2 t}(0, t) \theta_{2 x}(0, t) \\
& +k \theta_{1}(0, t) \theta_{1 t}(0, t)-\frac{\delta}{2} \int_{0}^{1}\left[\theta_{1 x}^{2}(x, t)+\theta_{1 t}^{2}(x, t)+\theta_{2 x}^{2}(x, t)+\theta_{2 t}^{2}(x, t)\right] \mathrm{d} x \\
& +\frac{\delta}{2}\left[\theta_{1 t}^{2}(1, t)+\theta_{1 x}^{2}(1, t)+\theta_{2 t}^{2}(1, t)+\theta_{2 x}^{2}(1, t)\right]-\frac{\delta}{2}\left[\theta_{1 x}^{2}(0, t)+\theta_{1 t}^{2}(0, t)+\theta_{2 x}^{2}(0, t)+\theta_{2 t}^{2}(0, t)\right] \\
& +\delta\left[\theta_{1 x}(1, t) \theta_{2 x}(1, t)-\theta_{1 x}(0, t) \theta_{2 x}(0, t)+\theta_{1 t}(1, t) \theta_{2 t}(1, t)-\theta_{1 t}(0, t) \theta_{2 t}(0, t)\right], \\
& =\left(\left(2+\frac{c^{2}}{2}\right) \delta-c\right) \theta_{1 t}^{2}(1, t)-\frac{\delta}{2} \int_{0}^{1}\left(\theta_{1 x}^{2}(x, t)+\theta_{1 t}^{2}(x, t)+\theta_{2 x}^{2}(x, t)+\theta_{2 t}^{2}(x, t)\right) \mathrm{d} x \\
& -\frac{\delta}{2}\left(k^{2} \theta_{1}^{2}(0, t)+\left(\theta_{1 t}(0, t)+\theta_{2 t}(0, t)\right)^{2}\right) .
\end{aligned}
$$

Let $\delta<\min \left\{1 / 3,(k / 3) 4 c / 4+c^{2}\right\}$ and $\alpha=\max \left\{1, k^{2}\right\}$ $\delta / 2 m_{1}$, and we get $\dot{V}(t) \leq-\alpha V(t)$, which gives the result.
Lemma 2. $\mathscr{B}$ is admissible to $e^{\mathscr{A} t}$.

Proof. By direct computation, we obtain

$$
\left\{\begin{array}{l}
\mathscr{A}^{*}\left(u_{1}, v_{1}, u_{2}, v_{2}\right)=\left(-v_{1},-u_{1}^{\prime \prime},-v_{2},-u_{2}^{\prime \prime}\right), \\
D\left(\mathscr{A}^{*}\right)=\left\{\left(u_{1}, v_{1}, u_{2}, v_{2}\right) \in H \cap\left(\mathscr{H}^{2}(0,1) \times \mathscr{H}^{1}(0,1)\right)^{2} \mid u_{1}^{\prime}(1)+u_{2}^{\prime}(1)=c v_{1}(1), u_{1}^{\prime}(0)=k u_{1}(0), u_{2}^{\prime}(0)=0, v_{1}=v_{2}\right\} .
\end{array}\right.
$$


The dual system of (10) is

$\begin{cases}\theta_{t t}^{*}(x, t)=\theta_{x x}^{*}(x, t), & 0<x<1, t\rangle 0, \\ \theta_{1}^{*}(1, t)=\theta_{2}^{*}(1, t), & t \geq 0, \\ \theta_{1 x}^{*}(1, t)+\theta_{2 x}^{*}(1, t)=c \theta_{1 t}^{*}(1, t), & t \geq 0, \\ \theta_{1 x}^{*}(0, t)=k \theta_{1}^{*}(0, t), & t \geq 0, \\ \theta_{2 x}^{*}(0, t)=0, & t \geq 0, \\ y(t)=-\frac{4-c^{2}}{4+q c} \theta_{1 t}^{*}(0, t) . & \end{cases}$

$\mathscr{A}$ generates a $C_{0}$-semigroup, so $\mathscr{A} *$ generates a $\mathrm{C}_{0}$-semigroup.

The energy of system (25) is

$$
\begin{aligned}
E(t)= & \frac{1}{2} \int_{0}^{1}\left(\theta_{1 x}^{*}(x, t)^{2}+\theta_{1 t}^{*}(x, t)^{2}+\theta_{2 x}^{*}(x, t)^{2}\right. \\
& \left.+\theta_{2 t}^{*}(x, t)^{2}\right) \mathrm{d} x+k \theta_{1}^{*}(0, t)^{2} .
\end{aligned}
$$

Then, $E(t) \leq E(0)$. Let $\rho(t)=\int_{0}^{1}(x-1) \theta_{1 x}^{*}(x, t) \theta_{1 t}^{*}(x, t)$ $+x \theta_{2 x}^{*}(x, t) \theta_{2 t}^{*}(x, t) \mathrm{d} x$. Differentiate $\rho(t)$ with respect to $t$ to yiel

$$
\begin{aligned}
\dot{\rho}(t)= & \frac{1}{2}\left[\theta_{1 x}^{* 2}(0, t)+\theta_{1 t}^{* 2}(0, t)+\theta_{2 x}^{* 2}(1, t)+\theta_{2 t}^{* 2}(1, t)\right] \mathrm{d} x \\
& -E(t)+\frac{1}{2} k \theta_{1}^{* 2}(0, t) .
\end{aligned}
$$

We, thus, have

$$
\begin{aligned}
\int_{0}^{T}|y(t)|^{2} \mathrm{~d} t & =\left(\frac{4-c^{2}}{4+q c}\right) \int_{0}^{2}\left|\theta_{1 t}^{*}(0, t)\right|^{2} \mathrm{~d} t \\
& \leq 2 T\left(\frac{\left(4-c^{2}\right)}{4+q c}\right)^{2} E(0)
\end{aligned}
$$

Similar to the computation of $A^{-1}$

$$
\begin{aligned}
& \mathscr{A}^{*-1}\left(\begin{array}{c}
u_{1} \\
v_{1} \\
u_{2} \\
v_{2}
\end{array}\right)=\left(\begin{array}{c}
(1+k x) a-\int_{0}^{x} v_{1}(\eta)(x-\eta) \mathrm{d} \eta \\
-u_{1}(x) \\
b-\int_{1}^{x} v_{2}(\eta)(x-\eta) \mathrm{d} \eta \\
-u_{2}(x)
\end{array}\right), \\
& \forall\left(\begin{array}{c}
u_{1} \\
v_{1} \\
u_{2} \\
v_{2}
\end{array}\right) \in \mathscr{H},
\end{aligned}
$$

where

$$
\begin{aligned}
a= & \frac{1}{k}\left[\int_{0}^{1}\left(\varphi_{1}(\eta)+\varphi_{2}(\eta)\right) \mathrm{d} \eta-c v_{1}(1)\right], \\
b= & (k+1) a-\int_{0}^{1} \varphi_{1}(\eta)(1-\eta) d \eta \\
& +\int_{0}^{1} \varphi_{2}(\eta)(1-\eta) \mathrm{d} \eta,
\end{aligned}
$$

$\mathscr{B}^{*} \mathscr{A}^{*-1}\left(\begin{array}{c}u_{1} \\ v_{1} \\ u_{2} \\ v_{2}\end{array}\right)=-u_{1}(0)$.

Therefore, on $\mathscr{H} \mathscr{B}^{*} \mathscr{A}^{*-1}$ is bounded. Combined with (26), we show that $\mathscr{B}$ is admissible to $e^{\mathscr{A} t}$ (see $[29,30]$ ).

From the two lemmas above, the evolution equation (14) has a unique weak solution in $\mathscr{H}$. Therefore, for $\forall\left(\theta_{1}(., t), \theta_{1 t}(., t), \theta_{2}(., t), \theta_{2 t}(., t)\right)^{T} \in \mathscr{H}, U_{0} \in L_{l o c}^{2}(0, \infty)$, system (10) has a unique weak solution $\left(\theta_{1}(x, t), \theta_{1 t}(x, t), \theta_{2}(x, t), \theta_{2 t}(x, t)\right)^{T} \in \mathscr{H}$, $\forall\left(u_{1}, v_{1}, u_{2}, v_{2}\right)^{T} \in D\left(\mathscr{A}^{*}\right)$,

$$
\begin{aligned}
\frac{\mathrm{d}}{\mathrm{d} t}\left\langle\left(\begin{array}{c}
\theta_{1} \\
\theta_{1 t} \\
\theta_{2} \\
\theta_{2 t}
\end{array}\right),\left(\begin{array}{c}
u_{1} \\
v_{1} \\
u_{2} \\
v_{2}
\end{array}\right)\right\rangle=\left\langle\left(\begin{array}{c}
\theta_{1} \\
\theta_{1 t} \\
\theta_{2} \\
\theta_{2 t}
\end{array}\right), \mathscr{A}^{*}\left(\begin{array}{c}
u_{1} \\
v_{1} \\
u_{2} \\
v_{2}
\end{array}\right)\right\rangle \\
\\
-g_{1}(0) \frac{4-c^{2}}{4+q c}\left[U_{0}(t)+r(t)\right],
\end{aligned}
$$


that is,

$$
\begin{aligned}
& \frac{\mathrm{d}}{\mathrm{d} t} \int_{0}^{1}\left[\theta_{1 x}(x, t) u_{1}^{\prime}(x)+\theta_{1 t}(x, t) v_{1}(x)+\theta_{2 x}(x, t) u_{2}^{\prime}(x)+\theta_{2 t}(x, t) v_{2}(x)\right] \mathrm{d} x \\
& =\int_{0}^{1}\left[-\theta_{1 x}(x, t) v_{1}^{\prime}(x)-\theta_{1 t}(x, t) u_{1}^{2}(x)-\theta_{2 x}(x, t) v_{2}^{\prime}(x)-\theta_{2 t}(x, t) u_{2}^{2}(x)\right] \mathrm{d} x \\
& \quad-v_{1}(0) \frac{4-c^{2}}{4+q c}\left[U_{0}(t)+r(t)\right] .
\end{aligned}
$$

Next, we will reject the disturbance $d$. An observer is chosen as

$$
\begin{aligned}
& y_{1}(t)=\int_{0}^{1}\left(\theta_{1 t}(x, t)+\frac{1}{2} c x \theta_{1 x}(x, t)+\theta_{2 t}(x, t)+\frac{1}{2} c x \theta_{2 x}(x, t)\right) \mathrm{d} x, t \geq 0, \\
& y_{2}(t)=\int_{0}^{1}\left(\theta_{1 t}(x, t)+\theta_{2 t}(x, t)\right) \mathrm{d} x, t \geq 0 .
\end{aligned}
$$

From equality (31), we have

$$
\dot{y}_{1}(t)=-\frac{c}{2} y_{2}(t)-\frac{4-c^{2}}{4+q c}\left(U_{0}+r(t)\right)
$$

We choose the high gain estimator to be (see [23])

$$
\left\{\begin{array}{l}
\dot{\hat{y}}(t)=-\frac{4-c^{2}}{4+q c}\left(U_{0}(t)+\hat{r}(t)\right)-\frac{c}{2} y_{2}(t)-\frac{1}{\varepsilon}\left(\hat{y}(t)-y_{1}(t)\right) \\
\dot{\hat{r}}(t)=\frac{1}{\varepsilon^{2}} \frac{4+q c}{4-c^{2}}\left(\hat{y}(t)-y_{1}(t)\right)
\end{array}\right.
$$

where $\varepsilon>0$ is the design small parameter and $\widehat{r}$ is the approximation of disturbance $r$. Let

$$
\tilde{y}(t)=\widehat{y}(t)-y_{1}(t), \tilde{r}(t)=\widehat{r}(t)-r(t),
$$

be the errors. Then, $\tilde{y}$ and $\tilde{r}$ satisfy

$$
\frac{\mathrm{d}}{\mathrm{d} t}\left(\begin{array}{c}
\tilde{y}(t) \\
\tilde{d}(t)
\end{array}\right)=\left(\begin{array}{cc}
-\frac{1}{\varepsilon} & -\frac{4-c^{2}}{4+q c)} \\
\frac{1}{\varepsilon^{2}} \frac{4+q c}{4-c^{2}} & 0
\end{array}\right)\left(\begin{array}{c}
\tilde{y}(t) \\
\tilde{d}(t)
\end{array}\right)+\left(\begin{array}{c}
0 \\
-1
\end{array}\right) \dot{d}(t)=A\left(\begin{array}{c}
\tilde{y}(t) \\
\tilde{d}(t)
\end{array}\right)+B \dot{d}(t)
$$
be

The collocated feedback controller of (10) is designed to where $\operatorname{sat}(x)=\left\{\begin{array}{l}M, x \geq M+1, \\ x, x \in(-M-1, M+1), \text { with controller } \\ -M, x \leq-M-1 .\end{array}\right.$ $U_{0}(t)=-\operatorname{sat}(\widehat{r}(t))$, 


$$
\begin{cases}\theta_{t t}\left(x, t=\theta_{x x}(x, t)\right), & 0<x<1, t>0, \\ \theta_{1}(1, t)=\theta_{2}(1, t), & t \geq 0, \\ \theta_{1 x}(1, t)+\theta_{2 x}(1, t)=c \theta_{1 t}(1, t), & t \geq 0, \\ \theta_{1 x}(0, t)=\frac{4-c^{2}}{4+q c}(-\operatorname{sat}(\widetilde{r}(t)+r(t))+r(t))+k \theta_{1}(0, t), & t \geq 0, \\ \theta_{2 x}(0, t)=0, & t \geq 0, \\ \widehat{y}(t)=-\frac{4-c^{2}}{4+q c}(-\operatorname{sat}(\widetilde{r}(t))+\widehat{r}(t))-\frac{c}{2} y_{2}(t)-\frac{1}{\varepsilon}\left(\hat{y}(t)-y_{1}(t)\right), & t \geq 0, \\ \widehat{r}(t)=\frac{1}{\mathcal{\varepsilon}^{2}} \frac{4-q c}{4+c^{2}}(\hat{y}(t)-y(t)), & t \geq 0 .\end{cases}
$$

We get the following theorem concerning the stability when disturbance in involved.
Theorem 1. If $|r|$ and $|\dot{r}|$ are bounded measurable, then, for any initial value $\theta(\cdot, t) \in \mathscr{H}$, there exists a unique solution $\left(\theta, \theta_{t}\right) \in C(0, \infty ; \mathscr{H})$ for the closed-loop system (39) of (7):

$$
\begin{cases}\theta_{t t}\left(x, t=\theta_{x x}(x, t)\right), & 0<x<1, t>0, \\ \theta_{1}(1, t)=\theta_{2}(1, t), & t \geq 0, \\ \theta_{1 x}(1, t)+\theta_{2 x}(1, t)=c \theta_{1 t}(1, t), & t \geq 0, \\ \theta_{1 x}(0, t)=\frac{4-c^{2}}{4+q c}(-s a t(\widetilde{r}(t)+r(t))+r(t))+k \theta_{1}(0, t), & t \geq 0, \\ \theta_{2 x}(0, t)=0, & t \geq 0, \\ \tilde{y}(t)=-\frac{1}{\varepsilon} \widetilde{y}(t)-\frac{4-c^{2}}{4+q c} \widetilde{r}(t), & t \geq 0, \\ \widetilde{r}(t)=\frac{1}{\varepsilon^{2}} \frac{4-q c}{4+c^{2}} \tilde{y}(t)-r(t), & t \geq 0, \\ y_{1}(t)=\int_{0}^{1}\left(\theta_{1 t}(x, t)+\frac{1}{2} c x \theta_{1 x}(x, t)+\theta_{2 t}(x, t)+\frac{1}{2} c x \theta_{2 x}(x, t)\right) \mathrm{d} x, & t \geq 0, \\ y_{2}(t)=\int_{0}^{1}\left(\theta_{1 t}(x, t)+\theta_{2 t}(x, t)\right) \mathrm{d} x, & \end{cases}
$$

Moreover, the solution of (39) converges to any arbitrary given neighborhood of zero when $t \longrightarrow \infty$ and $\varepsilon \longrightarrow 0$.

For the proof of Theorem 1, we omit the proof, since it can be easily proved followed by $[19,24]$.

\section{Numerical Simulations}

In this section, we compute the state for the closed-loop system (39) by the finite difference method [19]. Here, 


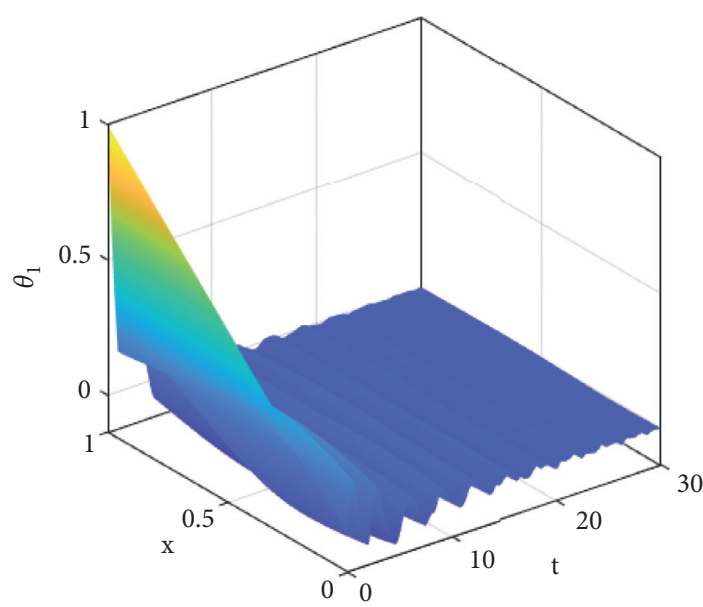

(a)

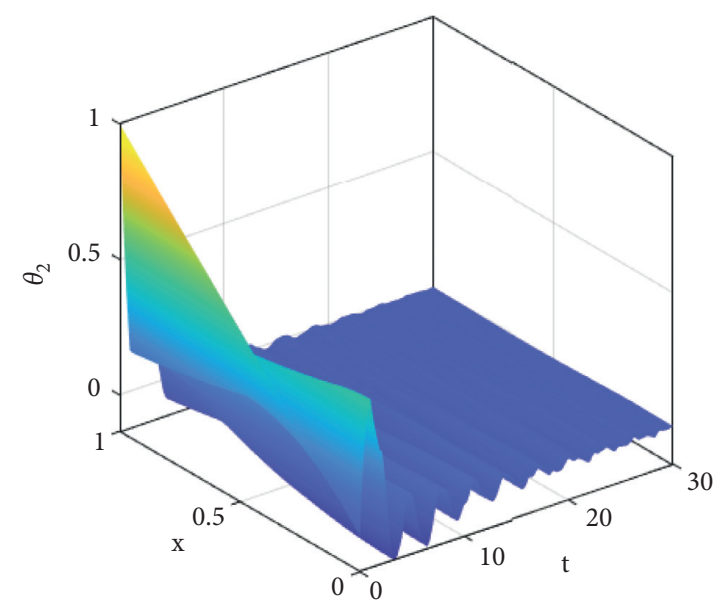

(b)

Figure 2: (a) The displacement of $\theta_{1}$; (b) the displacement of $\theta_{2}$.

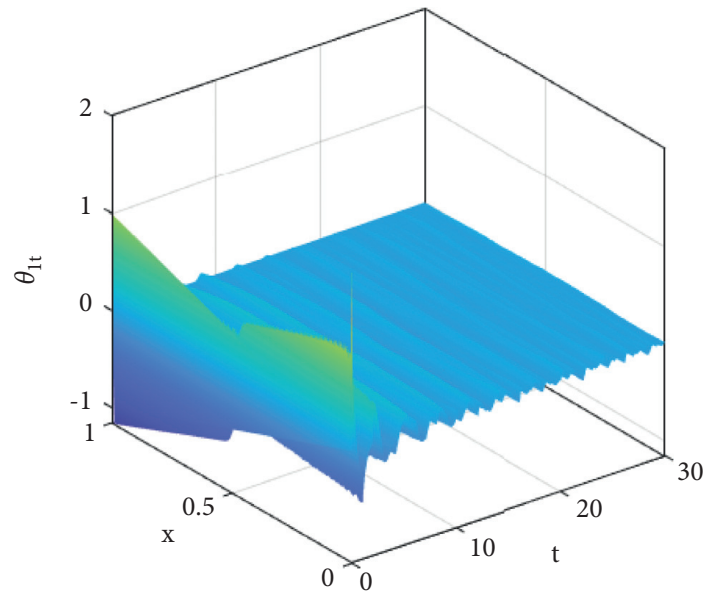

(a)

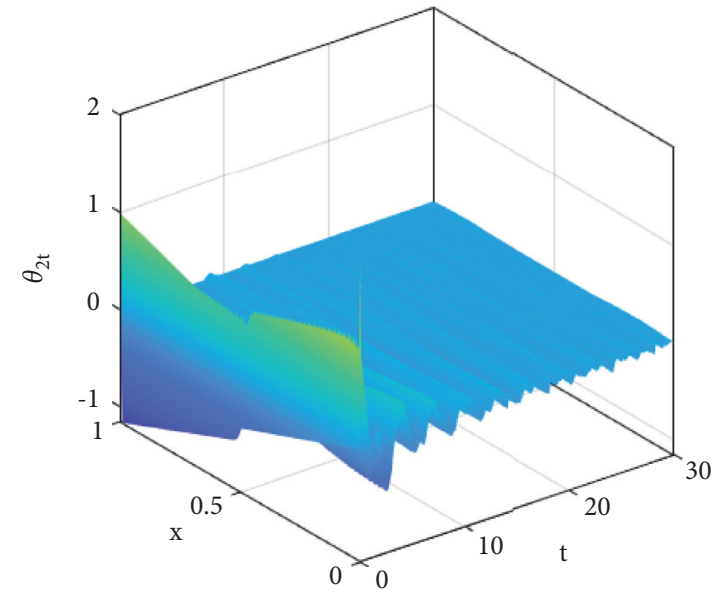

(b)

Figure 3: (a) The velocity of $\theta_{1 t}$; (b) the velocity of $\theta_{2 t}$.

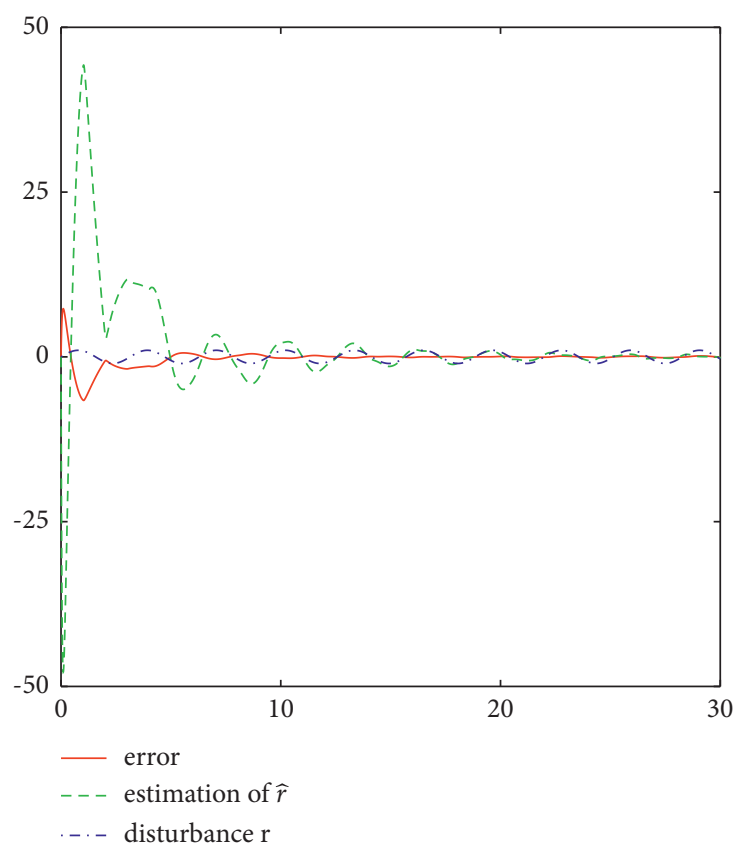

FIgURE 4: The displacement of $r, \widehat{r}$, and $\widetilde{r}$. 
$q=5, c=1.5, k=5$, and $d=3 \sin (2 t)$. Figure 2 shows that the displacement of $\theta_{1}$ and $\theta_{2}$ converges to zero. Figure 3 shows the velocity of $\theta_{1 t}$ and $\theta_{2 t}$ tend to zero. Figure 4 shows the estimation of $\hat{r}$ fits well with the value of $r$. Thus, the control law is effective.

\section{Conclusion}

A virtual connected string system with midway discontinue vertical force model is proposed for the underground multirobot system, and the Lyapunov-based stabilization control approach for virtual connected string is studied in the paper. The system is with Riemannian boundary. The backstepping is used to move the antistable term to the boundary thus to give the controller and Lyapunov approach is used to analyze the stability for the closed-loop system when the disturbance is absent. Then, ADRC is used to estimate and reject the disturbance. We can study the $n$ stings with discontinue vertical force in the further research.

\section{Data Availability}

The data used to support the findings of this study are included within the article.

\section{Conflicts of Interest}

The authors declare no conflicts of interest.

\section{Authors' Contributions}

Lingling $\mathrm{Su}$ and Lin Zhang conceived and designed the experiments; Xianhua Zheng and Shang Feng performed the experiments; Lin Zhang and Lingling Su built the pushing dynamics; Shang Feng and Yongshi Song analyzed the data; Lingling Su wrote the paper. Shang Feng translated and reedited the manuscript.

\section{Acknowledgments}

This work was supported by the National Natural Science Foundation of China (nos. 52004034 and 61903005), Science and Technology Research Program of Chongqing Municipal Education Commission (nos. KJQN202101413 and KJQN202001404), North China University of Technology Research Fund Program for Key Discipline (no. 110052972027/014), and North China University of Technology Scientific and technological innovation service building, Basic Science Research (no. 110052971921/102).

\section{References}

[1] L. Zhang, X. Zheng, S. Feng, and L. Su, "A noncommunicative memory-pushing fuzzy control strategy for sensorless multirobot systems," Complexity, vol. 2020, Article ID 7256427 , 15 pages, 2020.

[2] L. Zhang, X. Zheng, Q. Han, L. Su, and M. Luo, "A noncommunicative transportation approach for multiple physical connected objects," Intelligent Robotics and Applications, pp. 404-414, 2021.
[3] J. He, X. Liu, C. Mu, and C. Wang, "Hierarchical control of series-connected string converter-based islanded electrical power system," IEEE Transactions on Power Electronics, vol. 35, no. 1, pp. 359-372, 2019.

[4] H. Allouni, M. Kesri, and A. Benaissa, "On the asymptotic behaviour of two coupled strings through a fractional joint damper," Rendiconti del Circolo Matematico di Palermo Series 2, vol. 69, no. 2, pp. 613-640, 2020.

[5] B. Z. Guo and W. D. Zhu, "On the energy decay of two coupled strings through a joint damper," Journal of Sound and Vibration, vol. 203, no. 3, pp. 447-455, 1997.

[6] D. Benitez, J. Ungo, and H. Suarez, "Symmetry in the system of two blocks connected by a string with variable tension," Revista Cubana de Fisica, vol. 36, no. 2, p. 139, 2019.

[7] W. B. Qin and G. Orosz, "Experimental validation of string stability for connected vehicles subject to information delay," IEEE Transactions on Control Systems Technology, vol. 28, no. 4, pp. 1203-1217, 2020.

[8] A. Y. Khapalov, "Exponential decay for the one-dimensional wave equation with internal pointwise damping," Mathematical Methods in the Applied Sciences, vol. 20, no. 14, pp. 1171-1183, 1997.

[9] K.-S. Liu, "Energy decay problems in the design of a point stabilizer for coupled string vibrating systems," SIAM Journal on Control and Optimization, vol. 26, no. 6, pp. 1348-1356, 1988.

[10] Y. Lv, C. Li, X. K. Zhao, L. L. Li, and J. Li, “A novel approach for remanufacturing process planning considering uncertain and fuzzy information," Frontiers of Mechanical Engineering, vol. 16, no. 3, pp. 546-558, 2021.

[11] K. Li, F. Gao, S. B. Li, Y. Zhang, and H. B. Gao, "Robust cooperation of connected vehicle systems with eigenvaluebounded interaction topologies in the presence of uncertain dynamics," Frontiers of Mechanical Engineering, vol. 13, no. 3, pp. 354-367, 2018.

[12] R. Li, "Boundary exponential stabilization of a one-dimensional anti-stable wave equation with control matched disturbance," Engineering, vol. 12, no. 9, pp. 640-651, 2020.

[13] J. Liu, Y. Hu, B. Wu, K. Zhou, and M. F. Ge, "An adaptive sliding mode control technology for weld seam tracking," Frontiers of Mechanical Engineering, vol. 10, no. 1, pp. 95-101, 2015.

[14] J. J. Liu and J. M. Wang, "Active disturbance rejection control and sliding mode control of one-dimensional unstable heat equation with boundary uncertainties," IMA Journal of Mathematical Control and Information, 2015.

[15] J. W. Dong, C. D. Liang, M. F. Ge, T. F. Ding, and Z. W. Liu, "Finite-Time multi-target tracking of networked EulerLagrange systems via hierarchical active disturbance rejection control," International Journal of Control, 2021.

[16] B.-Z. Guo, H.-C. Zhou, A. S. AL-Fhaid, A. M. M. Younas, and A. Asiri, "Stabilization of Euler-Bernoulli beam equation with boundary moment control and disturbance by active disturbance rejection control and sliding mode control approaches," Journal of Dynamical and Control Systems, vol. 20, no. 4, pp. 539-558, 2014.

[17] R. Parvathy, A. E. Daniel, and C. M. Noufal, "Analysis of extended-state-observer and active-disturbance-rejectioncontrol in the speed control of DC motor system," Control and Intelligent Systems, vol. 47, no. 2, pp. 63-70, 2019.

[18] Z.-L. Zhao and B.-Z. Guo, "On active disturbance rejection control for nonlinear systems using time-varying gain," $E u$ ropean Journal of Control, vol. 23, pp. 62-70, 2015. 
[19] L. Su and J.-M. Wang, "Stabilisation of an anti-stable joint string with boundary disturbance," International Journal of Control, vol. 93, no. 5, pp. 1027-1038, 2020.

[20] J. Han, "From PID to active disturbance rejection control," IEEE Transactions on Industrial Electronics, vol. 56, no. 3, pp. 900-906, 2009.

[21] R. Wang, X. Li, and X. Ma, "Discrete reduced-order active disturbance rejection control for marine engines using variable sampling rate control scheme under limited bandwidth," IFAC-PapersOnLine, vol. 53, no. 2, pp. 1361-1366, 2020.

[22] B.-Z. Guo and F.-F. Jin, "The active disturbance rejection and sliding mode control approach to the stabilization of the Euler-Bernoulli beam equation with boundary input disturbance," Automatica, vol. 49, no. 9, pp. 2911-2918, 2013.

[23] B.-Z. Guo and F.-F. Jin, "Arbitrary decay rate for two connected strings with joint anti-damping by boundary output feedback," Automatica, vol. 46, no. 7, pp. 1203-1209, 2010.

[24] B.-Z. Guo and J.-J. Liu, "Sliding mode control and active disturbance rejection control to the stabilization of one-dimensional Schrödinger equation subject to boundary control matched disturbance," International Journal of Robust and Nonlinear Control, vol. 24, no. 16, pp. 2194-2212, 2014.

[25] M. Krstic and A. Smyshlyaev, "Boundary control of PDEs: a course on backstepping designs," Advances in Design and Control, Society for Industrial and Applied Mathematics (SIAM), Philadelphia, PA, USA, 2008.

[26] L. Su, W. Guo, J.-M. Wang, and M. Krstic, "Boundary stabilization of wave equation with velocity recirculation," IEEE Transactions on Automatic Control, vol. 62, no. 9, pp. 47604767, 2017.

[27] L. Su, S. Chen, J.-M. Wang, and M. Krstic, "Stabilization of a 2 $\times 2$ system of hyperbolic PDEs with recirculation in the unactuated channel," Automatica, vol. 120, Article ID 109147, 2020.

[28] A. Pazy, Semigroups of Linear Operators and Applications to Partial Differential Equations, Springer-Verlag, New York, NY, USA, 1983.

[29] G. Weiss, "Admissibility of unbounded control operators," SIAM Journal on Control and Optimization, vol. 27, no. 3, pp. 527-545, 1989.

[30] G. Weiss, "Admissible observation operators for linear semigroups," Israel Journal of Mathematics, vol. 65, no. 1, pp. 17-43, 1989. 